Y. Kitaoka

Nagoya Math. J.

Vol. 67 (1977), 159-164

\title{
SCALAR EXTENSION OF QUADRATIC LATTICES II
}

\author{
YOSHIYUKI KITAOKA
}

Let $k$ be a totally real algebraic number field, $\subseteq$ the maximal order of $k$, and let $L$ (resp. $M$ ) be a $Z$-lattice of a positive definite quadratic space $U$ (resp. $V$ ) over the field $\boldsymbol{Q}$ of rational numbers. Suppose that there is an isometry $\sigma$ from $\subseteq L$ onto $\subseteq M$. We have shown that the assumption implies $\sigma(L)=M$ in some cases in [2]. Our aim in this paper is to improve the results of [2]. In $\S 1$ we introduce the notion of $E$ type: Let $L$ be a positive definite quadratic lattice over $Z$. If any minimal vector of $L \otimes M$ is of the form $x \otimes y(x \in L, y \in M)$ for any positive definite quadratic lattice $M$ over $Z$, then we say that $L$ is of $E$-type. Some sufficient conditions for $E$-type are given in $\S 1$ and they are applied to our aim in $\S 2$.

Notations. As usual $Z$ (resp. $Q$ ) is the ring (resp. the field) of rational integers (resp. of rational numbers). By a positive definite quadratic lattice $L$ over $Z$ we mean a $Z$-lattice $L$ of a positive definite quadratic space $V$ over $\boldsymbol{Q}(\operatorname{rank} L=\operatorname{dim} V)$. For a positive definite guadratic lattice $L$ we denote $\min Q(x)$ by $m(L)$ where $Q$ is the quadratic form of $L$ and $x$ runs over non-zero elements of $L$, and we call an element $x$ of $L$ a minimal vector of $L$ if $Q(x)=m(L) . \quad Q(x), B(x, y)$ denote quadratic forms and corresponding bilinear forms $(2 B(x, y)=Q(x+y)$ $-Q(x)-Q(y))$.

§1. Let $L, M$ be positive definite quadratic lattices over $Z$ with bilinear forms $B_{L}, B_{M}$ respectively. Then the tensor product $L \otimes M$ over $Z$ can be made into a positive definite quadratic lattice over $Z$ with bilinear form $B$ such that $B\left(x_{1} \otimes y_{1}, x_{2} \otimes y_{2}\right)=B_{L}\left(x_{1}, x_{2}\right) B_{M}\left(y_{1}, y_{2}\right)$ for any $x_{i} \in L, y_{i} \in M$. Hereafter the tensor product $L \otimes M$ means this positive definite quadratic lattice over $Z$. Let $x$ (resp. $y$ ) be a minimal vector of $L$ (resp. $M$ ); then $x \otimes y \in L \otimes M$ implies $m(L \otimes M) \leq m(L) m(M)$. It is

Received September 10, 1976. 
known by Steinberg (p. 47 in [3]) that there is an example of $L, M$ such that $m(L \otimes M)<m(L) m(M)$.

DEFINITION. Let $L$ be a positive definite quadratic lattice over $Z$. We say that $L$ is of $E$-type if every minimal vector of $L \otimes M$ is of the form $x \otimes y(x \in L, y \in M)$ for any positive definite quadratic lattice $M$ over $Z$. Then $x$ (resp. $y$ ) is a minimal vector of $L$ (resp. $M$ ), and $m(L \otimes M$ ) is equal to $m(L) m(M)$.

Proposition 1. If $L_{1}, L_{2}$ are of E-type, ${ }^{*)}$ then $L_{1} \perp L_{2}, L_{1} \otimes L_{2}$ are of E-type.

Proof. Let $M$ be a positive definite quadratic lattice over $Z$. Let $v$ be a minimal vector of $\left(L_{1} \perp L_{2}\right) \otimes M$; then $v$ is of the form $x+y$ $\left(x \in L_{1} \otimes M, y \in L_{2} \otimes M\right)$. Since $x$ is orthogonal to $y$, we have $Q(v)=Q(x)$ $+Q(y)$. The minimality of $Q(v)$ yields $x=0$ or $y=0$. Hence $v$ is in $L_{1} \otimes M$ or $L_{2} \otimes M$, and $v$ is of the form $u \otimes w\left(u \in L_{1}\right.$ or $\left.L_{2}, w \in M\right)$. This means that $L_{1} \perp L_{2}$ is of $E$-type. Every minimal vector of $L_{1} \otimes L_{2}$ $\otimes M$ is of the form $x_{1} \otimes y$ where $x_{1}$ (resp. $y$ ) is a minimal vector of $L_{1}$ (resp. $\left.L_{2} \otimes M\right)$. As $y$ is of the form $x_{2} \otimes z\left(x_{2} \in L_{2}, z \in M\right)$, we have $x_{1} \otimes y=x_{1} \otimes x_{2} \otimes z$, and $x_{1} \otimes x_{2}$ is a minimal vector of $L_{1} \otimes L_{2}$. Hence $L_{1} \otimes L_{2}$ is of $E$-type.

Proposition 2. Let $L$ be of $E$-type. If a submodule $L_{1}$ of $L$ satisfies $m\left(L_{1}\right)=m(L)$, then $L_{1}$ is of E-type.

Proof. Let $M$ be a positive definite quadratic lattice over $Z$. Since we have $m(L) m(M)=m(L \otimes M) \leq m\left(L_{1} \otimes M\right) \leq m\left(L_{1}\right) m(M)=m(L) m(M)$, a minimal vector $v$ of $L_{1} \otimes M$ is one of $L \otimes M$. Hence $v$ is of the form $x \otimes y(x \in L, y \in M)$. As $y$ is primitive in $M, x$ is in $L_{1}$. Therefore $L_{1}$ is of $E$-type.

DEFinition. Let $n$ be a natural number. We put $\mu_{n}=\max \frac{m(A)}{\sqrt[n]{|A|}}$, where $A$ runs over positive definite real symmetric matrices with degree $n$, and $m(A)=\min _{x \in \boldsymbol{Z}^{n}-\{0\}}{ }^{t} x A x$.

LEMMA 1. If $n \geq 40$, then $\mu_{n}<\frac{n}{6}$.

*) When we say that $L$ is of $E$-type, $L$ is assumed to be a positive definite quadratic lattice over $Z$. 
Proof. It is known by [1] that

$$
\mu_{n}<\frac{2}{\pi} \Gamma\left(2+\frac{n}{2}\right)^{2 / n}
$$

Since $\Gamma(x)=\sqrt{2 \pi} x^{x-1 / 2} e^{-x+\mu(x)}\left(x>0, \mu(x)=\frac{\theta}{12 x}, 0<\theta<1\right)$, we have $\mu_{n}<\frac{2}{\pi}(2 \pi)^{1 / n}\left(2+\frac{n}{2}\right)^{1+3 / n} e^{-4 / n-1+1 / 3 n(n+4)}$. Put $f(x)=\log \frac{x}{6}-\log \left\{\frac{2}{\pi}(2 \pi)^{1 / x}\right.$ $\left.\left(2+\frac{x}{2}\right)^{1+3 / x} e^{-4 / x-1+1 / 3 x(x+4)}\right\}$. If $f(x)>0$ for $x \geq 40$, then Lemma is true.

Since $f(x)=\log x-\log 6-\log \frac{2}{\pi}-\frac{1}{x} \log 2 \pi-\left(1+\frac{3}{x}\right) \log \left(2+\frac{x}{2}\right)$ $+\frac{4}{x}+1-\frac{1}{3 x(x+4)}$, we get

$$
\begin{aligned}
x^{2} f^{\prime}(x)= & \log 2 \pi+3 \log \left(2+\frac{x}{2}\right)-3-\frac{4}{x+4} \\
& +\frac{2 x+4}{3(x+4)^{2}}>3 \log 22-3-\frac{1}{11}>0 \quad \text { if } x \geq 40 .
\end{aligned}
$$

Hence we have only to show $f(40)>0$. This is easy to see.

We denote by $\kappa$ the maximum of the number $k$ which satisfies that $\mu_{r} \geqq \sqrt{r}$ and $r \leq k$ imply $r=1$.

LEMMA 2. $\kappa$ is not smaller than 42.

Proof. It is known that $\mu_{n}(1 \leq n \leq 8)$ is $1, \sqrt{4 / 3}, \sqrt[3]{2}, \sqrt[4]{4}, \sqrt[5]{8}$, $\sqrt[6]{64 / 3}, \sqrt[7]{64}$, and 2 respectively. Hence $\kappa \geq 8$. Put

$$
g(x)=\log \frac{2}{\pi}(2 \pi)^{1 / x}\left(2+\frac{x}{2}\right)^{1+3 / x} e^{-4 / x-1+1 / 3 x(x+4)}-\log \sqrt{x} .
$$

Since $\log \mu_{n}-\log \sqrt{n}<g(n)$, we have only to show $g(x) \leq 0$ for $8 \leq x$ $\leq$ 42. Then $x^{2} g^{\prime}(x)=\frac{x}{2}-\log 2 \pi-3 \log \left(2+\frac{x}{2}\right)+3+\frac{4}{x+4}-\frac{2 x+4}{3(x+4)^{2}}$. Putting $h(x)=x^{2} g^{\prime}(x)$, we have

$$
\begin{aligned}
h^{\prime}(x) & =\frac{1}{2}-3 \frac{1}{x+4}-\frac{4}{(x+4)^{2}}-\frac{2}{3(x+4)^{2}}+\frac{4(x+2)}{3(x+4)^{3}} \\
& =\frac{1}{6(x+4)^{3}}\left(3 x^{3}+18 x^{2}-20 x-176\right) .
\end{aligned}
$$


Since $3 x^{3}+18 x^{2}-20 x-176>0$ for $x \geq 8$, we get $h^{\prime}(x)>0$. Moreover $h(8)$ is positive. Hence $g^{\prime}(x)$ is positive for $x \geq 8$. $g(42)<0$ is easy to see.

Remark. Rogers' result [5] may improve the number 42.

Lemma 3. Let $A, B$ be positive definite real symmetric matrices with degree $n$; then we have $\operatorname{Tr}(A B) \geq n \sqrt[n]{|A|} \sqrt[n]{|\bar{B}|}$.

Proof. Put $B=D[T]$ where $D$ is diagonal and $T$ is orthogonal. Let $a_{1}, \cdots, a_{n}$ and $d_{1}, \cdots, d_{n}$ be diagonals of $T A^{t} T, D$ respectively. Then

$$
\begin{aligned}
& \operatorname{Tr}(A B)=\operatorname{Tr}(A D[T])=\operatorname{Tr}\left(T A^{t} T D\right)=\sum a_{i} d_{i} \geq n \sqrt[n]{\prod\left(a_{i} d_{i}\right)}
\end{aligned}
$$

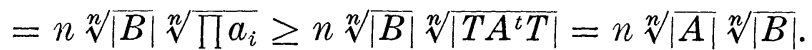

THEOREM 1. If $L$ is a positive definite quadratic lattice over $Z$ with $\operatorname{rank} L \leq \kappa$, then $L$ is of E-type.

Proof. Taking a positive definite quadratic lattice $M$ over $Z$, we put a minimal vector $v$ of $L \otimes M=\sum_{i=1}^{r} x_{i} \otimes y_{i}\left(x_{i} \in L, y_{i} \in M\right)$. In these representations of $v$ we take one with minimal $r$. Then $x_{1}, \cdots, x_{r}$ and $y_{1}, \cdots, y_{r}$ is linearly independent in $L, M$ respectively. Noting $Q(v)$ $=Q\left(\sum x_{i} \otimes y_{i}\right)=\sum_{i, j} B\left(x_{i}, x_{j}\right) B\left(y_{i}, y_{j}\right)=\operatorname{Tr}\left(\left(B\left(x_{i}, x_{j}\right)\left(B\left(y_{i}, y_{j}\right)\right)\right.\right.$, we get $Q(v) \geq r\left(\left|\left(B\left(x_{i}, x_{j}\right)\right) \|\left(B\left(y_{i}, y_{j}\right)\right)\right|\right)^{1 / r}$ by Lemma 3 . On the other hand $Q(v)$ $=m(L \otimes M) \leq m(L) m(M) \leq m\left(Z\left[x_{1}, \cdots, x_{r}\right]\right) m\left(Z\left[y_{1}, \cdots, y_{r}\right]\right)$. Therefore

$$
r \leq \frac{m\left(Z\left[x_{1}, \cdots, x_{r}\right]\right)}{\left|\left(B\left(x_{i}, x_{j}\right)\right)\right|^{1 / r}} \frac{m\left(Z\left[y_{1}, \cdots, y_{r}\right]\right)}{\left|\left(B\left(y_{i}, y_{j}\right)\right)\right|^{1 / r}} \leq \mu_{r}^{2} .
$$

By the definition of $\kappa$ we have $r=1$. This completes the proof.

Remark. In the Steinberg's example for $m(L \otimes M)<m(L) m(M)$, rank $L \geq 292$.

THEOREM 2. Let $L$ be a positive definite quadratic lattice over $\boldsymbol{Z}$. If $m(L) \leqq 6$, and the discriminant $d L_{0}$ of any non-zero submodule $L_{0}$ of $L$ is not smaller than 1 , then $L$ is of E-type.

Proof. Let $M$ be a positive definite quadratic lattice over $Z$, and let a minimal vector $v$ of $L \otimes M$ be $\sum_{i=1}^{r} x_{i} \otimes y_{i}$. As in the proof of Theorem 1 we may assume that $x_{1}, \cdots, x_{r}$, and $y_{1}, \cdots, y_{r}$ are linearly independent in $L, M$ respectively. Put $L_{0}=Z\left[x_{1}, \cdots, x_{r}\right]$ and $M_{0}=Z\left[y_{1}\right.$, $\left.\cdots, y_{r}\right]$. Then $m(L \otimes M)=Q(v) \geq r \sqrt[r]{d L_{0}} \sqrt[r]{d M_{0}} \geq r \sqrt[r]{d M_{0}}$. On the other 
hand $m(L \otimes M) \leq m(L) m(M) \leq 6 m\left(M_{0}\right)$. Hence we get $r / 6 \leq m\left(M_{0}\right) / \sqrt[r]{d M_{0}}$ $\leq \mu_{r}$. Lemma 1 implies $r \leq 40$, and Lemma 2 implies that $L_{0}$ is of $E$-type. Since $v \in L_{0} \otimes M_{0}$ and $m(L \otimes M) \leq m\left(L_{0} \otimes M_{0}\right), v$ is a minimal vector of $L_{0} \otimes M_{0}$. Therefore $v$ is of the form $x \otimes y\left(x \in L_{0}, y \in M_{0}\right)$, and this completes the proof.

§. We apply the results of $\S 1$ to our problem. Some other applications will appear in the forthcoming paper.

In this section $E$ denotes a totally real algebraic number field with degree $n$, and $\mathfrak{D}$ is the maximal order of $E$. From Theorem III of p. 2 in [6] follows that $\operatorname{tr}_{E / Q} a^{2} \geq n$ for any non-zero element $a$ of $\mathfrak{D}$, and moreover the equality yields $a= \pm 1$. Let $L$ be a positive definite quadratic lattice over $Z$; then we denote by $\subseteq L$ the tensor product of $\subseteq$ and

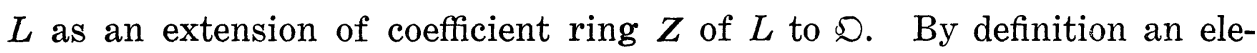
ment $v$ of $\subseteq L$ gives the rational minimum of $\subseteq L$ if and only if $Q(v)$ $=\min Q(u)$ where $u$ runs over a non-zero element of $D L$ with $Q(u) \in \boldsymbol{Q}$. When we regard $\mathcal{O}$ as a positive definite quadratic lattice over $Z$ with the bilinear form $B(x, y)=\operatorname{tr}_{E / Q} x y$, we write $\bar{D}$ instead of $\mathfrak{D}$.

LEMma. Let $L$ be a positive definite quadratic lattice over $Z$. If $\mathfrak{D}$ or $L$ is of E-type, then a vector of $\bigcirc L$ which gives the rational minimum of $\subseteq L$ is already in $L$.

Proof. As indicated in the introduction $B$ denotes the bilinear form of $L$. We define a new bilinear form $\tilde{B}$ on $\subseteq L$ which is defined by $\tilde{B}(x, y)=\operatorname{tr}_{E / Q} B(x, y) \quad(x, y \in \subseteq L)$. This quadratic lattice is denoted by

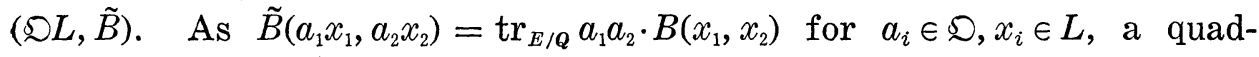
ratic lattice $(\subseteq L, \tilde{B})$ is isometric to $\tilde{D} \otimes L$. Take a vector $v$ of $\subseteq L$ which gives the rational minimum of $\subseteq L$; then we have

$$
0 \neq \tilde{B}(v, v)=n Q(v) \leq n m(L)=m(\tilde{D}) m(L)=m(\tilde{D} \otimes L)=m((§ L, \tilde{B})) .
$$

Hence $v$ is a minimal vector of $(\subseteq L, \tilde{B})$. Regarding $v$ as an element of $\tilde{\mathfrak{D}} \otimes L$, we get $v=a \otimes x(a \in \mathfrak{D}, x \in L)$, where $a$ is a minimal vector of $\tilde{\mathfrak{D}}$, and so $a= \pm 1$. This implies $v \in L$.

THEOREM. Let $L, M$ be positive definite quadratic lattices over $\boldsymbol{Z}$. Assume that rank $L \leq \kappa$ or $\tilde{\mathfrak{D}}$ is of E-type. Then, for any isometry $\sigma$ from $\subseteq L$ on $\subseteq M$ over the ring $D$, we get $\sigma(L)=M$. 
Proof. Lemma implies that a vector $v$ of $L$ which gives the rational minimum of $\subseteq L$ is in $L$, and $\sigma(v)$ is also in $M$ since $\sigma(v)$ gives the rational minimum of $\subseteq M$. Therefore $\sigma$ induces an isometry from $\subseteq v^{\perp}$ on $\Im \sigma(v)^{\perp}$. Inductively we get $\sigma(\boldsymbol{Q} L)=\boldsymbol{Q} M . \quad \sigma(囚 L)=\subseteq M$ yields $\sigma(L)=M$.

Remark 1. If $n \leq \kappa$ or $n / m \leq 6$ where $m Z=\left\{\operatorname{tr}_{E / Q} a ; a \in \mathfrak{D}\right\}(m>0)$, then Theorem 1,2 in $\S 1$ imply that $\tilde{D}$ is of $E$-type.

Remark 2. Assume that $E=E_{1} E_{2}$ where $E_{i}$ is a totally real algebraic number field with maximal order $\mathfrak{D}_{i}$. Moreover we assume that $\left(d E_{1}, d E_{2}\right)=1$ and $\widetilde{\mathfrak{D}}_{i}$ is of $E$-type $(i=1,2)$. Then $\widetilde{\mathfrak{D}} \cong \widetilde{\mathfrak{D}}_{1} \otimes \widetilde{\mathfrak{D}}_{2}$ is of $E$ type.

\section{REFERENCES}

[1] H. F. Blichfeldt, The minimum value of quadratic forms, and the closest packing of spheres, Math. Ann., 101 (1929), 605-608.

[2] Y. Kitaoka, Scalar extension of quadratic lattices, Nagoya Math. J., 66 (1977), 139-149.

[ 3 ] J. Milnor-D. Husemoller, Symmetric bilinear forms, Springer-Verlag, 1973.

[ 4 ] O. T. O'Meara, Introduction to quadratic forms, Springer-Verlag, 1963.

[5] C. A. Rogers, Packing and covering, Cambridge University Press, 1964.

[6] C. L. Siegel, Gasammelte Abhandlungen III, Springer-Verlag, 1966. 